\title{
Role of Nitric Oxide Synthase Family in Diabetic Neuropathy
}

\section{Constantina Heltianu ${ }^{1 *}$ and Cristian Guja ${ }^{2}$}

${ }^{1}$ Institute of Cellular Biology and Pathology "N. Simionescu”, Bucharest, Romania

${ }^{2}$ Institute of Diabetes, Nutrition and Metabolic Diseases "Prof. NC Paulescu", Bucharest, Romania

\begin{abstract}
Diabetes mellitus is one of the most common chronic diseases and a major health problem in nearly all countries. Its prevalence has risen sharply worldwide during the past few decades. The increase in its prevalence is largely due to an epidemic of type 2 diabetes (T2DM) but also the type 1 diabetes (T1DM) incidence is rising all over the world. The "classical" microvascular complications of both types of diabetes are represented by diabetic retinopathy, diabetic renal disease, and diabetic polyneuropathy (DPN). The latter has known to be heterogeneous regarding symptoms, pattern of neurologic involvement, and progression in time, pathologic alterations, and underlying mechanisms. DPN results from the direct damage of peripheral nerve components and of the associated vasa nervorum. The main contributor to the DPN development is the cumulative effect of chronic hyperglycemia - duration of diabetes and level of metabolic control. Apart the major role of hyperglycemia one of the most important factor is nitric oxide (NO) system. The NO production and local release are dramatically changed and contribute to the DPN development. The process takes place by the modulation of the nitric oxide synthase (NOS) enzymes responsible for NO synthesis by the diabetic milieu. While the decline in NO bioavailability is due to the decrease in neuronal (NOS1) and endothelial (NOS3) expression, the increase of local NO levels with the formation of peroxynitrites arises from the upregulation of inducible (NOS2) protein. Thus, NOS genes became natural candidates for the study of DPN genetics. In comparison with the other diabetes microvascular complications, data regarding the genetic background of DPN are rather scarce, particularly for NOS genes. The aim of this review is to present the pathogenesis of DPN in both type 1 and type 2 diabetes and the effect of the NOS genes-NOS enzymes-NO system.
\end{abstract}

Keywords: Type 1 diabetes; Type 2 diabetes; Diabetic neuropathy; Nitric oxide system; NOS gene polymorphisms

Abbreviations: T1DM: Type 1 Diabetes; T2DM: Type 2 Diabetes; DN: Diabetic Neuropathy; DPN: Diabetic Polyneuropathy; DSPN: symmetrical, length-dependent Diabetic Sensorimotor Poly Neuropathy; DAN: Diabetic Autonomic Neuropathy; ROS: Reactive Oxygen Species; PARP: Poly ADP-Ribose Polymerase; PKC: Protein Kinase C; AGE: Advanced Glycation End-products; AKR1B1: Aldose Reductase Gene; GWL: Genome Wide Scan Linkage; GWA: Genome Wide Scan Association; NO: Nitric Oxide; RBC: Red Blood Cells; NOx: Stable end-product Nitrite/Nitrate; NOS1: Neuronal Nitric Oxide Synthase; NOS2: Inducible Nitric Oxide Synthase; NOS3: Endothelial Nitric Oxide Synthase; SNP: Single Nucleotide Polymorphism; rs 1799983: The $G$ to $T$ polymorphism at position 894 in exon 7 of NOS3 gene; $r$ 2070744: The substitution from $T$ to $C$ at the position -786 in the promoter region of NOS3 gene.

\section{Introduction}

Diabetes mellitus is one of the most prevalent chronic diseases of modern societies and a major health problem in nearly all countries. Its prevalence has risen sharply worldwide during the past few decades [1]. Moreover, predictions for the next 20 years show that diabetes prevalence will continue to rise, reaching epidemic proportions by 2030: 439 million adults worldwide representing 7.7\% of the total world population [1]. This increase in the prevalence is largely due to the epidemic of obesity and consequent type 2 diabetes (T2DM), but type 1 diabetes (T1DM) incidence is also rising in all developing countries [2].

Both T1DM and T2DM are characterized by the slow progression towards the generation of some specific lesions of the blood vessels affecting both small (microangiopathy) and larger (macroangiopathy) vessels. The "classical" microvascular complications of diabetes are represented by diabetic retinopathy (the main cause of blindness in adults), diabetic renal disease, representing currently the main cause of renal substitution therapy (dialysis or renal transplantation) in developed countries, and diabetic neuropathy. Major epidemiological and interventional studies showed that chronic hyperglycemia is the main contributor to diabetic tissue damage $[3,4]$. Increasing evidence suggests that oxidative stress and consequent endothelial dysfunction may be the key mediators of the deleterious effects of hyperglycemia. If hyperglycemia remains the main risk factor for the development of diabetic chronic complications, an important contribution can be attributed to genetic risk factors, some of them common for all microvascular complications (diabetic retinopathy, neuropathy, and renal disease) and some specific for each of them [5]. Additional factors are represented by some accelerators such as hemodynamic (hypertension) and metabolic (insulin resistance, dyslipidemia) components.

One of the key factors with a crucial role in vascular physiopathology is nitric oxide (NO), on which hyperglycemia has a major impact. Its production depends on the whole family of nitric oxide synthase (NOS) enzymes expressed in many cell types of the peripheral nervous system [6-8]. In the following pages, we present the pathogenesis of diabetic neuropathy in both T1DM and T2DM with emphasis on the effects of the various NO system components (NOS genes-NOS enzymes-NO).

${ }^{*}$ Corresponding author: Constantina Heltianu, Institute of Cellular Biology and Pathology "N. Simionescu", 8, B. P. Hasdeu Street, Bucharest - 050568, Romania Tel: (004021) 31927 37; Fax: (004021) 31945 19; E-mail: ina.heltianu@icbp.ro Received October 03, 2011; Accepted November 20, 2011; Published November 24, 2011

Citation: Heltianu C, Guja C (2011) Role of Nitric Oxide Synthase Family in Diabetic Neuropathy. J Diabetes Metab S5:002. doi:10.4172/2155-6156.S5-002

Copyright: (c) 2011 Heltianu C, et al. This is an open-access article distributed under the terms of the Creative Commons Attribution License, which permits unrestricted use, distribution, and reproduction in any medium, provided the original author and source are credited. 


\section{Diabetic Neuropathy}

\section{Definition, clinical signs, morphological changes}

Diabetic neuropathy (DN) is a chronic diabetes complication present in both two major phenotypes: T1DM and T2DM. It is a chronic microvascular complication affecting both somatic and autonomic peripheral nerves. It may be defined as the presence of symptoms and/or signs of peripheral nerve dysfunction in people with diabetes, after the exclusion of other causes of neuropathy $[5,9]$. Many neuropathic patients have signs of neurological dysfunction upon clinical examination (decreased sensation for pain, temperature, vibration etc.) but have no symptoms (negative symptoms neuropathy). One the contrary, some patients have positive symptoms (burning, itching, freezing, pain, often with nocturnal exacerbations), usually with distal onset and proximal progression. Frequently, patients have signs of autonomic neuropathy such as resting tachycardia, postural hypotension, vomiting, diarrhea and poor glycemic control following gastrointestinal neuropathy, bladder dysfunction, erectile dysfunction, etc. Most often, DN represents an insidious and progressive disorder which begins with a long asymptomatic stage. Regarding the morphological changes, characteristic are aspects of primary segmental demyelination of long axons, axonal degeneration, decrease density of small unmyelinated fibers, paranodal anarchic regeneration processes, and Waller's degeneration [10].

\section{Classification of DN}

$\mathrm{DN}$ is known to be heterogeneous regarding symptoms, pattern of neurologic involvement, pathologic alterations, underlying mechanisms and progression in time [11,12]. Therefore, the term of $\mathrm{DN}$ describes a heterogeneous group of disorders with a wide range of abnormalities. Many classifications of DN were proposed over time $[12,13]$.

According to the 2005 ADA classification [13], DN can be separated into generalized symmetric polyneuropathies and focal/multifocal varieties (including multiple mononeuropathy, lumbosacral, thoracic and cervical radiculoplexus neuropathies). By far the most prevalent are the generalized symmetric polyneuropathies, usually designated as DPN. On its turn, DPN can be further divided into typical, symmetric sensorimotor polyneuropathy (DSPN) and atypical DPN.

DSPN is a length-dependent disease induced by metabolic dysfunction of nerve fibers combined with perineural microvessel alterations, both being the result of chronic hyperglycemia and cardiovascular hemodynamic risk covariates. The co-existence of other diabetic microvascular complications (retinopathy and nephropathy) in the same patient strongly suggests that DSPN is directly attributable to diabetes. However, other causes of sensorimotor polyneuropathy need to be excluded in order to make a positive diagnosis $[12,13]$.

Atypical DPN is different from DSPN regarding the onset, evolution, clinical manifestations, pathogenic mechanisms, etc. and has been less well characterized and studied $[12,13]$. The most important phenotypes are represented by the painful DPN and the diabetic autonomic neuropathy (DAN). DAN may affect the cardiovascular, gastrointestinal and urogenital systems as well as the sudomotor function.

\section{Prevalence of DN}

Correct estimates of the DPN prevalence are hard to obtain due to objective factors. For example, the diagnosis of the "negative symptoms patients" can be made only by active screening, usually with complex investigations such as nerve conduction velocity or evoked potential measurements. All these explain why the currently reported prevalence data vary widely. In T1DM, the EURODIAB study estimated the prevalence of DPN to be $\sim 25 \%$ in Europe, with a maximum of $\sim 70 \%$ in Romania [14]. For T2DM, the mean prevalence of DPN is estimated at $30-50 \%$, increasing with diabetes duration $[12,15]$. The diagnosis of subclinical DAN requires also complex investigations and its prevalence was reported to reach up to $90 \%$ in T2DM subjects [13].

DPN also appears to be more common in males than in females and in T2DM than T1DM. Thus, we previously used two separate groups of Romanian subjects with T1DM (211 patients) and T2DM (250 patients) for genetic assessments [16,17]. In this patient cohort the prevalence of DPN (alone) was significantly higher $(\mathrm{P}<0.001)$ in T2DM as compared with T1DM subjects (Figure 1).

\section{Pathogenesis of DN}

Despite the substantial progresses made in unveiling the molecular mechanisms characterizing the pathogenesis of DPN, this is still incompletely understood. Current data suggest that DPN results from the damage of the vasa nervorum (microvessels responsible for the irrigation of neural tissue) associated with the direct damage of neuronal components. Both are the result of the interaction between metabolic (chronic hyperglycemia, dyslipidemia, oxidative stress), hemodynamic/ischemic (microangiopathy of the nerve blood vessels) factors and impairment of the nerve fiber repair mechanisms [10]. The vascular and metabolic mechanisms act simultaneously and have an additive effect. The presence of neuronal microangiopathy is the main reason for considering DPN as one of the chronic microvascular complications of diabetes.

The cumulative effect of chronic hyperglycemia (duration of diabetes and level of metabolic control) is the main contributor to the DPN development [18]. Chronic hyperglycemia contributes to the development of DPN by the same mechanism involved in all other diabetic microvascular complications. An unifying mechanism was proposed by Brownlee et al. in 2000, suggesting that the key element is the hyperglycemia induced overproduction of superoxide anions $\left(\mathrm{O}^{-}\right)$ by the mitochondrial electron transport chain $[19,20]$. It was postulated that hyperglycemia induces increased mitochondrial production of reactive oxygen species (ROS) followed by nuclear DNA strand breaks that, in turn, activate the enzyme poly ADP-ribose polymerase (PARP) leading to a cascade process that finally activates four major pathways of diabetic complications:

(1) Increased aldose reductase activity and activation of the polyol pathway leading to increased sorbitol accumulation with osmotic effects, including neuronal "edema", impaired nerve conduction and, finally, apoptosis [10]

(2) Activation of protein kinase $\mathrm{C}$ (PKC) with subsequent activation of NF-kB pathway

(3) Intracellular advanced glycation end-products (AGE) generation

(4) Activation of the hexosamine pathway [19]. As for DPN, several studies showed that enhanced oxidative stress in peripheral nerves and vasa nervorum leads to neural dysfunction, impaired neurotrophic support as well as characteristic neuropathic morphologic nerve alterations [21].

Apart the major role of hyperglycemia, additional risk factors for DPN are represented by increasing age, alcohol consumption or other drug abuse and classic cardiovascular risk factors including 
hypertension, dyslipidemia, obesity, cigarette smoking and albuminuria [18]. In addition, genetic susceptibility has an important contribution in determining the global risk for DPN. Several of the cardiovascular risk factors reported to be associated with DPN are markers of insulin resistance. The potential link between insulin resistance and diabetes microvascular complications including DPN is their association with oxidative stress and endothelial dysfunction. In addition, one of the most important links between the metabolic and vascular mechanisms of DPN was reported to be the depletion of nitric oxide and failure of antioxidant protection, both resulting in increased oxidative stress [10]. Thus, the nitric oxide synthase genes became natural candidates for the study of DPN genetics.

\section{Genetic background of DN}

Genetically, diabetic neuropathy is a complex disorder emerging from the balance between multiple predisposing and protective gene variants as well as following the interaction with environmental factors. In comparison with the other chronic diabetes microvascular complications, data regarding the genetic background of DPN are rather scarce. To our best knowledge, no genome wide scans (GWL or GWA) were performed for the identification of DPN genes. In the same time, several studies regarding the effect of some candidate genes were published, but these included usually only small number of patients/ controls and few of them were replicated in other, independent datasets. However, a few consistent data can be retrieved from the literature.

One of the genes studied for association with diabetic neuropathy is the aldose reductase gene (AKR1B1) on chromosome $7 \mathrm{q} 35$. Thus, a case/control study of white T1DM patients suggested that the $Z+2$ allele of the (AC)n microsatellite of AKR1B1 might have a protective effect against the development of DPN [22]. More recently, the genotyping of a white T1DM population indicated that the presence of the AKR1B1 $Z-2$ allele significantly increases the risk for autonomic neuropathy [23]. Unfortunately, no hard data confirmed the role of $A K R 1 B 1$ gene variants in T2DM neuropathy patients [5].

Other significant (but usually not reconfirmed) associations with the risk for DPN were reported for variants of the poly ADP-ribose polymerase (PARP-1) gene on chromosome 1q42, uncoupling protein $U C P 2$ and UCP3 genes on chromosome 11, the genes encoding the antioxidant proteins catalase and superoxide dismutase and the gene encoding the neuronal $\mathrm{Na}^{+} / \mathrm{K}^{+}$-ATPase [5]. Data regarding the involvement of nitric oxide synthase genes will be provided in the following pages.

\section{Nitric oxide effects in DPN}

In peripheral nerves, the production of nitric oxide (NO), the main vasorelaxant of the vasomotor function, depends on the expression of all nitric oxide synthases: constitutive neuronal NOS1, endothelial NOS3 and NOS3-like from red blood cells (RBC) and inducible NOS2. According to the local NO concentration, this factor may have beneficial or harmful effects. In physiological concentrations its positive effects are mediated by activation of cyclic GMP [24]. The deleterious actions (e.g. impairment of microvascular vasorelaxation) take place in case of: (i) low NO amounts when the effects are mediated independent of cyclic GMP due to its defective mechanism [25], leading to low levels of plasma NO2/NO3 (NOx), and (ii) high NO concentrations (also independent of cyclic GMP) when insulin impairs the NO ability to generate its messenger, followed by an increase in plasma NOx [26]. In the latter case, a reaction between $\mathrm{NO}$ and superoxide anions (another key mediator of glucose-induced oxidative injury) takes place with the formation of peroxynitrites (ONOO-). The latter, rapidly causes protein nitration or nitrosylation, lipid peroxidation, deoxyribonucleic acid (DNA) damage, and cell death $[24,27,28]$.

\section{NO derived from constitutive NOS enzymes}

NOS1-derived NO mediates the nitrergic neurotransmission by a major non-adrenergic non-cholinergic pathway in many organs of the urogenital, gastrointestinal and cardiovascular systems, to control local vasomotor tone and blood flow. The nitrergic nerves surrounding the organs are able to release small quantities (picomoles) of NOS1-derived NO which antagonizes the effect of contractile neurotransmitters (e.g. noradrenaline), a process extremely strong in favor of $\mathrm{NO}$ [29]. A recent report showed the presence of NOS1 in the vascular endothelium, suggesting that this isoform is expressed in many cell types of the peripheral nervous system, including vasa nervorum [7].

In both types of diabetes, the NOS1-derived NO has a low local level, inhibits vasodilatation and causes nerve ischemia with axonal degeneration [30]. This process was proposed to take place in two phases in T1DM. In the early stages of diabetes, NOS1 expression in the nitrergic axons decreases, and insulin treatment is able to reverse the process. Two explanations were suggested: the first, a similar NO reduction in the body cell of nitrergic neurons (not experimentally confirmed), and the second, more likely, a defect in the axonal transport of NOS1 enzyme from the body cell to the axons. Further, in the second phase, in parallel with diabetes progression, the neurons lose completely their NOS1 content and suffer apoptotic degenerative changes which are irreversible, without any response to insulin treatment. In both phases the level of NO released from axons and then from neurons decreases progressively and the nitrergic relaxation is significantly altered [29].

NOS3-derived NO is produced locally also in small quantities (picomoles) as in the case of NOS1, it causes a tonic vasodilator effect, and it is generally accepted that it has an important role in maintenance of normal nerve vascular tonus. It is presumed that NOS1-derived NO has a much higher contribution to blood pressure regulation than NOS3-derived NO [29]. The mediator has a dual function, depending mainly on the monomeric or dimeric form of NOS3. The dimeric form is involved in NO synthesis, and when the dimmer/monomer ratio is reduced (as in the diabetic milieu), the shift towards the monomeric NOS3 form contributes to the increased cellular oxidative stress, followed by the decline in NO bioavailability, a process which might contribute to the development of DPN [31].

Analysis of tissues and large arteries of type 1 and type 2 diabetic animals indicated both increases and decreases in NOS3 expression. The microvasculature was less studied. Regarding the peripheral nerve system, from the endothelial cells of epineurial and endoneurial blood vessels (vasa nervorum), those from the endoneurium which surrounds individual axons seem to be particularly vulnerable. So, the endoneurial changes and then the impairment of microvascular vasorelaxation by reductions in NOS3 mediated NO production represent events which start very early, even before overt hyperglycemia (the prediabetic state), as reported in both human and experimental T2DM models $[8,32]$.

To obtain a full picture of NO involvement in DPN, one has to consider also the red blood cells (RBC) which represent a major deposit for NO. An important role has been attributed to RBC in the critical balance between the generation and scavenging of $\mathrm{NO}$ which determines its local bioavailability and influences vasomotor control [33]. It was reported that on the internal layer of the RBC membrane there is a 
NOS isotype with activity and regulatory mechanisms very similar with those of NOS3. This enzyme has essential regulatory functions for RBC deformability [34]. In diabetes, these cells are affected when decreased deformability, increased membrane microviscosity and $\mathrm{RBC}$ aggregation take place [35]. Reduced RBC deformability leads to decreased peripheral neural tissue blood flow followed by endoneurial hypoxia when the nerve energy supply is compromised.

\section{NO derived from inducible NOS enzyme}

NOS2-derived NO is synthesized and released in large quantities (nanomoles) under certain conditions (e.g. hyperglycemia), and it becomes neurotoxic (via peroxynitrite formation) for the local neural tissue, interfering with vasa nervorum control and leading to deterioration of regional circulation. So the action of the constitutive NOS enzymes seem to be compromised $[28,32,36]$. The immunological NOS2 isoform was found to be highly expressed in demyelinating environments when the excess of NO directly inhibits the axonal mitochondrial functions through oxidative modifications of the mitochondrial proteins, a process implicated in the pathogenesis of DPN [6]. Prolonged exposure of axons to NO triggers acute neuronal conduction block. The neurotoxic NO effect on electrically active axons is only transient, but even a brief period of focal energy restriction can trigger Waller's degeneration [37]

\section{Nitric oxide synthase genes and DPN}

NOS1: The human gene encoding NOS1 is localized on chromosome 12q14, and the transcription unit of 29 exons spans a region greater than $240 \mathrm{~kb}$. Different polymorphisms of NOS1 were described, but to our knowledge, there is only one report in which a NOS1 polymorphism was analyzed for association with DPN. The CA repeat in the 3'-UTR region (exon 29) of NOS1 was found not to be a risk factor for DPN in T1DM [38]. Multiple transcript variants that differ in the 5' UTR have been also described, but the full-length nature of these transcripts is not known. The alternative promoters contain several putative transcription factor binding sites (e.g. for Sp-1, AP1, NFkB) which permit cell-, tissue-, and site-specific transcriptional regulation of NOS1 activity. Due to the differences in the N-terminus protein structure, three NOS1 protein variants $(\alpha, \beta$, and $\gamma)$ were reported and NOS1 $\alpha$, which contains a specific $\mathrm{N}$-terminus protein interaction domain (PDZ) required for homodimerization into the active configuration, is the unique functionally isoform [39].

NOS2A: Human NOS2A gene located on chromosome 17q11.2-12 has the transcription start site in exon 2 and the stop codon in exon 27. This gene encodes NOS2 protein which has two different functional catalytic enzyme domains: the oxygenase domain encoded by exons 1 to 13 , and the reductase domain encoded by exons 14 to 27 . A large number of polymorphisms have been identified in this gene. Data for the relationship of NOS2A polymorphisms with DPN were reported for two microsatellite repeats in the promoter region, the biallelic insertion (AAAAT)/deletion (AAAT) repeat [40], and the (CCTTT) (n) repeat with nine alleles [38]. The latter might affect $N O S 2 A$ transcription followed by an alteration of NOS2 activity and local NO level responsible for the susceptibility or/and severity of the disease. In T1DM, carriers of 14-(CCTTT) repeat allele have lower risk of DPN $[38,41]$. In T2DM, a significant association between DPN and the $A A A T$ repeat insertion allele (which confers higher NOS2 expression than the deletion variant) was reported. However, when diabetic nephropathy was introduced into the predicting model for DPN, the latter becomes marginally significant [40].
NOS3: NOS3 gene is located on chromosome $7 \mathrm{q} 36.1$ and it comprises 26 exons spanning approximately $21 \mathrm{~kb}$ of genomic DNA [42]. Several polymorphisms have been reported in NOS3 promoter, exon and intron regions [9]. The most studied variant from the promoter region was the single nucleotide polymorphism (SNP) at position -786 represented by a base substitution from $T$ to $C(r s 2070744)$. In previous studies it was shown that individuals with $-786 \mathrm{C}$ allele had a reduced activity of the NOS3 gene promoter (explained by the fact that a DNA binding protein, the replication protein $\mathrm{A} 1$, has the ability to bind only to the $-786 \mathrm{C}$ allele isoform), resulting in a $\sim 50 \%$ reduced NOS3 transcription, with the subsequent decrease in both protein expression and serum NOx levels [43].

A polymorphism of NOS3 in intron $4(4 a / 4 b)$ is based on a variable 27-base pair tandem repeat; consisting of four (allele $4 a$ ), five (allele $4 b$ ) or six (allele $4 c$ ) repeats. Previous studies have suggested that deletion of one of the five nucleotide repeats in intron 4 could affect the NOS 3 transcription and processing rates, thus resulting in modulation of NOS3 enzymatic activity and, apparently, affecting plasma NOx concentrations, with the potentiality of the $4 a / 4 a$ genotype to have an effect on microangiopathy later on in the diabetes evolution $[44,45]$. Carriers of the $4 a$ allele were found exhibiting $\sim 20 \%$ lower NOx levels than $4 b / 4 b$ homozygous subjects. The regulation of NOS3 expression is more complicated considering the strong linkage of $4 a / 4 b$ variant with $r s 2373961$ and $r s 2070744$ when the $4 b / b$ genotype might act independently and in coordination with the other variants [46]

Among polymorphisms found in NOS3 exons, the SNP at position 894 ( $G$ to $T$ ) in exon 7 ( $r$ s1799983) was also studied. It was reported that this variant changes the NOS3 protein sequence, probably leading to an alteration of enzyme activity [47], and controls the NOS3 intracellular distribution, interacting with proteins that mediate its degradation [48].

The previous few reports which analyzed the influence of NOS3 polymorphisms on DPN were only performed in T1DM subjects. Data from Caucasian patients with T1DM genotyped for rs2070744, $r s 1799983$ and $4 b / 4 a$ showed that none of these polymorphisms were associated with DPN. Other T1DM report revealed that in the group with the lowest incidence of confirmed distal symmetric polyneuropathy (DSPN), the highest proportion of $894 G G$ homozygotes of rs 1799983 was among the subjects with this microvascular disorder [38,41,47,4950].

To our knowledge, we were the first to analyze the relationship between NOS3 polymorphisms and DPN in the same population for the two types of diabetes in separate cohorts [16,17]. As shown in (Figure 1), the differences between T1DM vs. T2DM regarding the frequency of diabetes complications were the prevalence of DPN and combined microvascular complications. Analysis of allele frequencies of NOS3 $4 b / 4 a$ and $r s 1799983$ variants showed that in patients without any microvascular complications, while the proportion of the $4 a$ allele (Figure 2, A) was higher $\left(\chi^{2}=3.08 ; \mathrm{P}=0.079\right)$ in T2DM as compared with T1DM, the $894 \mathrm{~T}$ allele (Figure 2, B) was significantly higher $\left(\chi^{2}\right.$ $=8.98 ; \mathrm{P}=0.003)$ in T1DM vs T2DM. The allele differences of NOS 3 polymorphisms in DPN or combined complications in T1DM vs. T2DM were comparable.

Previously, we reported that the NOS3 $4 a / 4 a$ genotype does not represent a risk factor for T1DM-DPN [16]. When the T1DM subjects were divided in the three subgroups (free complications, DPN only, and combined complications, represented by 28,20 , and 77 patients, respectively) the $4 \mathrm{a}$ allele carriers were significantly higher in combined 


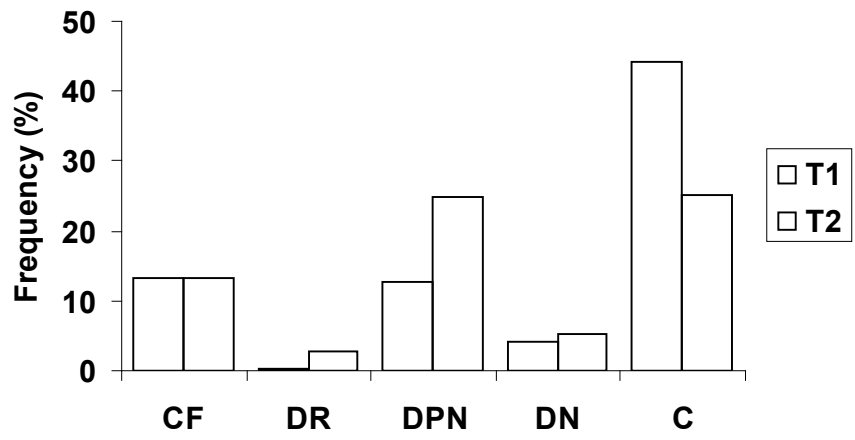

Figure 1: Frequencies of microvascular complications in type 1 and type 2 diabetes. CF, free microvascular complications; DR, diabetic retinopathy; DPN, diabetic neuropathy; DN, diabetic nephropathy; C, combined diabetic microvascular complications (retinopathy, neuropathy, nephropathy. Note, the high proportion of DPN ( 2 fold) in T2DM.

2A

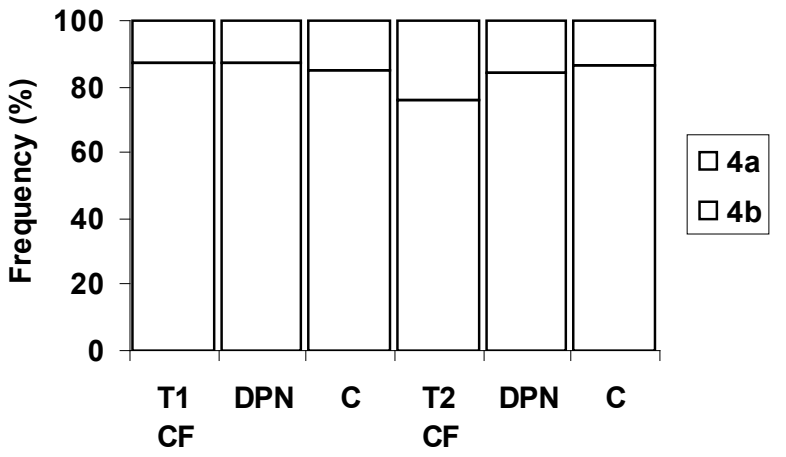

2B

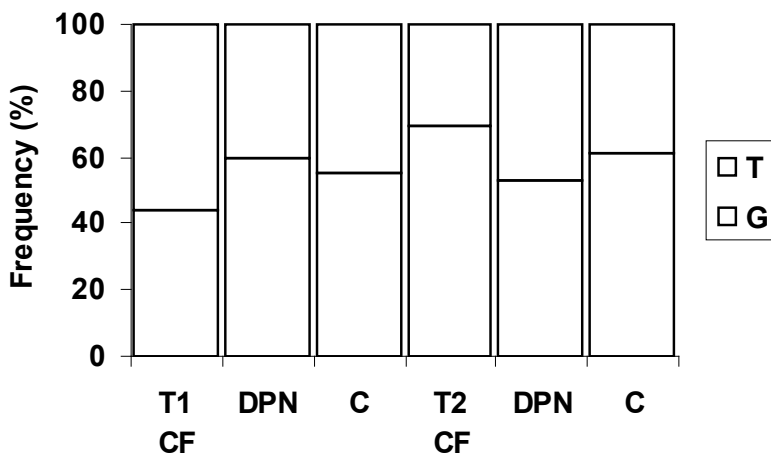

Figure 2: Allele frequencies of NOS34b/4a (A) and NOS3 rs1799983 (B) in type 1 and type 2 diabetic-associated microvascular complications. In (Figure $2 A$ ) the alleles $4 a$ and $4 b$ correspond to the rare and wild type, respectively, and in (Figure $2 \mathrm{~B}$ ), the alleles $\mathrm{T}$ and $\mathrm{G}$ signify the rare and wild type, respectively. CF, free microvascular complications; DPN, diabetic neuropathy; C, combined diabetic microvascular complications (retinopathy, neuropathy, nephropathy)

complications ( $\mathrm{P}=0.001)$ as compared with DPN (Table 1), suggesting that the $4 a$ allele carriers with DPN had a possible higher risk for diabetic retinopathy or/and nephropathy. We were not able to analyze separately the data for diabetic retinopathy and nephropathy due to the very small number of cases (see Figure 1). However, other reports indicated that the NOS3 $4 b / 4 a$ was associated with diabetic nephropathy [44], and not related with diabetic retinopathy or its severity [50]; so, we can speculate that in the case of our data, the $4 a$ allele might have an effect on the development of diabetic nephropathy in those subjects with DPN.

For T2DM, a significantly higher proportion of $4 \mathrm{a}$ allele carriers was found in free complications patients than in DPN, but the possible protective role of the $4 a$ allele for the low prevalence of DPN was at the border of statistical significance (Table 1). Choosing the obese T2DM patients only, this relationship was significant as we reported recently [17]. A recent study on T2DM Iranian patients reported a significant association between NOS3 $4 b / 4 a$ polymorphism and DPN when the authors compared DPN patients with healthy non-diabetic volunteers, but the association was no longer significant when DPN patients were compared with DPN-negative subjects [51], a finding that was in agreement with our data for the whole population of T2DM subjects. Subjects genotyped for NOS3 rs 1799983 variant and analyzed separately for the two types of diabetes indicated a significant correlation of the $894 T$ allele with DPN as compared with free complication patients with T2DM (Table 1), a result previously reported for the obese patients [17].

Finally, in view of the fact that NO is produced by all NOS isotypes with specific locations in the peripheral nerve system, we considered important to analyze all their gene variants together, although the reported data are rather limited (Table 2). In T1DM most reported data showed a non specific association of NOS variants with DPN [38,41], except for the 14-CCTTT NOS2A allele and $894 \mathrm{~T}$ allele of NOS3 rs1799983 which seem to be disease-protective polymorphisms $[16,38,41]$. We recently reported that, in T1DM patients without microvascular complications, the plasma NO metabolite levels of the 894TT homozygotes were very close to those of non-diabetic subjects [16], suggesting indirectly that NOS2- and NOS3-derived $\mathrm{NO}$ were in the normal range, strengthening the possible protective role of the respective polymorphisms. The relationship between these polymorphisms and plasma NO metabolite levels or expression of the respective NOS enzyme still raises controversy.

\begin{tabular}{|c|c|c|c|c|c|c|}
\hline \multirow{2}{*}{$\begin{array}{l}\text { NOS3 } \\
\text { gene }\end{array}$} & \multirow{2}{*}{ Disease } & & \multicolumn{2}{|l|}{ T1DM } & \multicolumn{2}{|l|}{ T2DM } \\
\hline & & & Wild & Rare & Wild & Rare \\
\hline \multirow{6}{*}{$4 b / 4 a$} & CF & \multirow{3}{*}{$\begin{array}{l}\text { alleles } \\
(\%)\end{array}$} & 87.50 & 12.50 & 75.81 & 24.19 \\
\hline & DPN & & 87.50 & 12.50 & 84.26 & 15.74 \\
\hline & C & & 85.06 & 14.94 & 86.27 & 13.73 \\
\hline & CF/DPN & \multirow{3}{*}{$\begin{array}{l}\text { P Value } \\
\left(x^{2}\right)\end{array}$} & \multicolumn{2}{|c|}{$1.00(0.00)$} & \multicolumn{2}{|c|}{$0.17(1.84)$} \\
\hline & CF/C & & \multicolumn{2}{|c|}{$0.66(0.20)$} & \multicolumn{2}{|c|}{$0.09(2.90)$} \\
\hline & DPN/C & & \multicolumn{2}{|c|}{$0.001(10.20)$} & \multicolumn{2}{|c|}{$0.68(0.17)$} \\
\hline \multirow{6}{*}{$\begin{array}{l}\text { rs1799983 } \\
\text { (G894T) }\end{array}$} & CF & \multirow{3}{*}{$\begin{array}{l}\text { alleles } \\
(\%)\end{array}$} & 44.23 & 55.77 & 69.35 & 30.65 \\
\hline & DPN & & 60.00 & 40.00 & 52.88 & 47.12 \\
\hline & C & & 55.19 & 44.81 & 61.11 & 38.89 \\
\hline & CF/DPN & \multirow{3}{*}{$\begin{array}{l}\text { P Value } \\
\left(X^{2}\right)\end{array}$} & \multicolumn{2}{|c|}{$0.13(2.25)$} & \multicolumn{2}{|c|}{$0.037(4.36)$} \\
\hline & CF/C & & \multicolumn{2}{|c|}{$0.17(1.87)$} & \multicolumn{2}{|c|}{$0.28(1.16)$} \\
\hline & DPN/C & & \multicolumn{2}{|c|}{$0.59(0.30)$} & \multicolumn{2}{|c|}{$0.23(1.46)$} \\
\hline
\end{tabular}

NOS3, endothelial nitric oxide synthase; CF, free microvascular complications DPN, diabetic neuropathy; C, combined diabetic microvascular complications (retinopathy, neuropathy, nephropathy). Significance (P Value) was 0.05 or less as accepted. Note, in T1DM the number of NOS3 4a carriers was significantly higher in $\mathrm{C}$ as compared with DPN subjects, suggesting that the rare allele was a risk factor for the prevalence of more than one microvascular disorder. In T2DM NOS3 $894 T$ allele might have an important role in the prevalence of DPN as the rare allele was significantly higher as compared with $\mathrm{CF}$.

Table 1: Allele frequencies of NOS3 $4 b / 4 a$ and NOS3 rs1799983 in diabetic neuropathy. 


\begin{tabular}{|c|c|c|c|c|c|}
\hline Gene & Variant & $\begin{array}{lr}\mathrm{N} & \mathrm{O} \\
\text { association }\end{array}$ & $\begin{array}{l}\text { DPN } \\
\text { risk }\end{array}$ & DPN protection & Reference \\
\hline \multicolumn{6}{|c|}{ Type 1 diabetes } \\
\hline NOS1 & CA repeats & $P>0.05$ & & & 38,41 \\
\hline NOS $2 A$ & CCTTT repeats & & & Tendency & 38,41 \\
\hline \multirow[b]{3}{*}{ NOS3 } & $4 a / 4 b$ & $P>0.05$ & & & $16,38,41$ \\
\hline & \multirow[b]{2}{*}{$\begin{array}{l}\text { rs1799983 } \\
\text { (G894T) }\end{array}$} & $P>0.05$ & & & 38,41 \\
\hline & & & & $\begin{array}{l}P<0.05 \\
\text { for } 894 T\end{array}$ & 16,46 \\
\hline \multicolumn{6}{|c|}{ Type 2 diabetes } \\
\hline NOS2A & $A A A A T / A A A T$ & & $\begin{array}{l}\mathrm{P}<0.05 \\
\text { for } A A A A T\end{array}$ & & 40 \\
\hline NOS3 & $4 a / 4 b$ & & & $\begin{array}{l}P<0.05^{*} \\
\text { for } 4 a\end{array}$ & 17 \\
\hline NOS3 & $\begin{array}{l}\text { rs1799983 } \\
\text { (G894T) }\end{array}$ & & $\begin{array}{l}P<0.05 \\
\text { for } 894 T\end{array}$ & & 17 \\
\hline
\end{tabular}

NOS1, neuronal nitric oxide synthase; NOS2A, inducible nitric oxide synthase NOS3, endothelial nitric oxide synthase; $\mathrm{P}$, significance was 0.05 or less as accepted; * obese T2DM patients

Table 2: Gene polymorphisms of nitric oxide synthases in diabetic neuropathy, cumulative reports.

Interestingly, in T2DM, the same 894 T allele of NOS3 rs1799983 [17], and the $A A A A T$ (insertion) NOS2A allele [40] were associated with DPN risk (Table 2). In addition, we found a significant diseaseprotective role of the $4 a$ NOS 3 allele. Knowing that $4 a / 4 b$ NOS 3 variant is apparently haplotype-dependent, and the haplotype containing $894 T / 4 a$ represents a risk for diabetes-associated microvascular disorders [51-53], we can speculate that both the AAAAT NOS2A and NOS3 rs 1799983 polymorphisms might be involved in disease development. Nevertheless, additional data to confirm these findings are needed.

\section{Conclusion}

All over the world the diabetes will reach epidemic proportions in the near future. While the cumulative effect of diabetes duration and degree of metabolic control represents the main contributor to the DPN development, the genetic risk factors are important contributors to the disease severity. For a good functionality of the peripheral nerve system, the normal compartmentalization of NOS enzymes to provide physiological local NO levels seems to be crucial. Although we know most of the DPN tissue targets where NOS isotypes are localized, their individual contributions as well as the interrelationship between them to the diabetes-induced injury and the development of degenerative changes in both somatic and autonomic peripheral nerves remain a debate problem. Interaction between metabolic and haemodynamic factors as well as susceptibility of peripheral nerves to NO system indicate a very complex and multifactorial phenomenon which takes place in DPN. The main issue is the management of changes which occur in the early stages of diabetes, a period when it was reported that they are reversible, before the appearance of the structural damages when the process seems to be without return. Genetic prediction and use of individual aetiological processes, as well as the translation of recent molecular knowledge into potential therapeutic targets will contribute selectively to the preventive and therapeutic interventions in this complex disease.

\section{Acknowledgments}

This work was supported in part by grants of collaboration with the National
Institute of Diabetes, Nutrition and Metabolic Diseases "Prof. NC Paulescu", obtained from the Romanian Academy, and Ministry of Education and Research and Innovation (BIOTECH Program).

\section{References}

1. Shaw JE, Sicree RA, Zimmet PZ (2010) Global estimates of the prevalence of diabetes for 2010 and 2030. Diabetes Res Clin Pract 87: 4-14.

2. Vehik K, Dabelea D (2011) The changing epidemiology of type 1 diabetes: why is it going through the roof? Diabetes Metab Res Rev 27: 3-13.

3. DCCT Research Group (1993) The effect of intensive treatment of diabetes on the development and progression of long-term complications in insulindependent diabetes mellitus. The Diabetes Control and Complications Tria Research Group. N Engl J Med 329: 977-986.

4. UK Prospective Diabetes Study (UKPDS) Group (1998) Intensive blood glucose control with sulphonylureas or insulin compared with conventional treatment and risk of complications in patients with type 2 diabetes (UKPDS 33). Lancet 352: 837-853

5. Cimponeriu D, Crăciun AM, Apostol P, Radu I, Guja C, et al. (2010) The genetic background of diabetes chronic complications: Genetics of diabetes. The truth unveiled. Cheța D (Ed), Acad Rom \& S. Karger AG, Bucharest/Basel, chapter 4: 193-334.

6. Kiryu-Seo S, Ohno N, Kidd GJ, Komuro H, Trapp BD (2010) Demyelination increases axonal stationary mitochondrial size and the speed of axonal mitochondrial transport. J Neurosci 30: 6658-6666.

7. Vareniuk I, Pacher P, Pavlov IA, Drel VR, Obrosova IG (2009) Periphera neuropathy in mice with neuronal nitric oxide synthase gene deficiency. Int $J$ Mol Med 23: 571-580

8. Yamagishi S, Matsui T (2011) Nitric oxide, a janus-faced therapeutic target for diabetic microangiopathy_Friend or foe? Pharmacol Res 64: 187-194.

9. Heltianu C, Guja C, Manea S-A (2011) Genetic determinants of microvascula complications in type 1 diabetes: Type 1 Diabetes - Pathogenesis, Genetics and Immunotherapy, InTech Open Access Publisher, ISBN 978-953-307-3620 , Book edited by Prof. David Wagner, in print.

10. Boulton AJ (2007) Diabetic neuropathy: classification, measurement and treatment. Curr Opin Endocrinol Diabetes Obes 14: 141-145.

11. Dyck PJ, Thomas PK, Llewelyn JG, Tomlinson DR, Thomas PK (2005) Diabetic neuropathies: Peripheral neuropathy. (4thedn), Dyck PJ, Thomas PK (Eds) Elsevier company, Philadelphia, pp. 1951-1992.

12. Tesfaye S, Boulton AJ, Dyck PJ, Freeman R, Horowitz M, et al. (2010) Diabetic neuropathies: update on definitions, diagnostic criteria, estimation of severity, and treatments. Diabetes Care 33: 2285-2293.

13. Boulton AJ, Vinik AI, Arezzo JC, Bril V, Feldman EL, et al. (2005) Diabetic neuropathies: a statement by the American Diabetes Association. Diabetes Care 28: 956-962.

14. EURODIAB Prospective Complications Study Group (2005) Vascular risk factors and diabetic neuropathy. N Engl J Med 352: 341-350.

15. Vinik Al, Mehrabyan A (2004) Diabetic neuropathies. Med Clin North Am 88: 947-999.

16. Heltianu C, Manea SA, Guja C, Robciuc A, lonescu-Tîrgovişte C (2009) Polymorphism in exon 7 of the endothelial nitric oxide synthase gene is associated with low incidence of microvascular damage in type 1 diabetic neuropathy. Cent Eur J Biol 4: 521-527.

17. Manea SA, Robciuc A, Guja C, Heltianu C (2011) Identification of gene variants in NOS3, ET-1 and RAS that confer risk and protection against microangiopathy in type 2 diabetic obese subjects. Biochem Biophys Res Commun 407: 486490

18. Tesfaye S, Chaturvedi N, Eaton SE, Ward JD, Manes C, et al. (2005) Vascular risk factors and diabetic neuropathy. N Engl J Med 352: 341-350.

19. Brownlee M (2005) The pathobiology of diabetic complications. An unifying mechanism. Diabetes 54: 1615-1625.

20. Nishikawa T, Edelstein D, Du XL, Yamagishi S, Matsumura T, et al. (2000) Normalizing mitochondrial superoxide production blocks three pathways of hyperglycaemic damage. Nature 404: 787-790.

21. Hounsom L, Corder R, Patel J, Tomlinson DR (2001) Oxidative stress 
Citation: Heltianu C, Guja C (2011) Role of Nitric Oxide Synthase Family in Diabetic Neuropathy. J Diabetes Metab S5:002. doi:10.4172/2155-6156. S5-002

participates in the breakdown of neuronal phenotype in experimental diabetic neuropathy. Diabetologia 44: 424-428

22. Heesom AE, Millward A, Demaine AG (1998) Susceptibility to diabetic neuropathy in patients with insulin dependent diabetes mellitus is associated with a polymorphism at the $5^{\prime}$ end of the aldose reductase gene. J Neurol Neurosurg Psychiatry 64: 213-216.

23. Donaghue KC, Margan SH, Chan AK, Holloway B, Silink M, et al. (2005) The association of aldose reductase gene (AKR1B1) polymorphisms with diabetic neuropathy in adolescents. Diabet Med 22: 1315-1320.

24. González-Hernández T, Rustioni A (1999) Expression of three forms of nitric oxide synthase in peripheral nerve regeneration. J Neurosci Res 55: 198-207.

25. Cameron NE, Cotter MA (2007) Erectile dysfunction and diabetes mellitus: Mechanistic considerations from studies in experimental models. Curr Diabetes Rev 3: 149-158.

26. Piatti PM, Monti LD, Zavaroni I, Valsecchi G, Van Phan C, et al. (2000) Alterations in nitric oxide/cyclic-GMP pathway in nondiabetic siblings of patients with type 2 diabetes. J Clin Endocrinol Metab 85: 2416-2420.

27. Cowell RM, Russell JW (2004) Nitrosative injury and antioxidant therapy in the management of diabetic neuropathy. J Investig Med 52: 33-44.

28. Vareniuk I, Pavlov IA, Obrosova IG (2008) Inducible nitric oxide synthase gene deficiency counteracts multiple manifestations of peripheral neuropathy in a streptozotocin-induced mouse model of diabetes. Diabetologia 51: 2126-2133.

29. Cellek S (2004) Point of NO return for nitrergic nerves in diabetes: A new insight into diabetic complications. Curr Pharm Des 10: 3683-3695.

30. Keswani SC, Bosch-Marcé M, Reeda N, Angela Fischer, Gregg L Semenza et al. (2011) Nitric oxide prevents axonal degeneration by inducing HIF-1dependent expression of erythropoietin. Proc Natl Acad Sci USA 108: 49864990.

31. Labinskyy N, Hicks S, Grijalva J, Edwards J (2010) The contrary impact of diabetes and exercise on endothelial nitric oxide synthase function. Webmedcentral Article ID: WMC001376.

32. Cameron NE, Cotter MA (2008) Pro-inflammatory mechanisms in diabetic neuropathy: focus on the nuclear factor kappa B pathway. Curr Drug Targets 9 : 60-67.

33. Ulker P, Yaras N, Yalcin O, Celik-Ozenci C, Johnson PC, et al. (2011) Shear stress activation of nitric oxide synthase and increased nitric oxide levels in human red blood cells. Nitric Oxide 24: 184-191.

34. Kleinbongard P, Schulz R, Rassaf T, Lauer T, Dejam A, et al. (2006) Red blood cells express a functional endothelial nitric oxide synthase. Blood 107: 29432951.

35. Kunt T, Schneider S, Pfützner A, Goitum K, Engelbach M, et al. (1999) The effect of human proinsulin C-peptide on erythrocyte deformability in patients with type I diabetes mellitus. Diabetologia 42: 465-471.

36. Toda N, Imamura T, Okamura T (2010) Alteration of nitric oxide-mediated blood flow regulation in diabetes mellitus. Pharmacol Ther 127: 189-209.

37. Alvarez S, Moldovan M, Krarup C (2008) Acute energy restriction triggers Wallerian degeneration in mouse. Exp Neurol 212: 166-178.

38. Zotova EV, Voron'ko OE, Bursa TR, Galeev IV, Strokov IA, et al. (2005) Polymorphic markers of the NO synthase genes and genetic predisposition to diabetic polyneuropathy in patients with type 1 diabetes mellitus. Mol Bio (Mosk) 39: 224-229.

39. Savidge TC (2011) S-nitrosothiol signals in the enteric nervous system: lessons learnt from big brother. Front Neurosci 5: 31

40. Morris BJ, Markus MA, Glenn CL, Adams DJ, Colagiuri S, et al. (2002) Association of a functional inducible nitric oxide synthase promoter variant with complications in type 2 diabetes. J Mol Med 80: 96-104.
41. Nosikov VV (2004) Genomics of type 1 diabetes mellitus and its late complications. Mol Biol (Mosk) 38: 150-164

42. Marsden PA, Heng HHQ, Scherer SW, Stewart RJ, Hall AV, et al. (1993) Structure and chromosomal localization of the human constitutive endothelial nitric oxide synthase gene. J Biol Chem 268: 17478-17488.

43. Taverna MJ, Elgrably F, Selmi H, Selam JL, Slama G (2005) The T-786C and C774T endothelial nitric oxide synthase gene polymorphisms independently affect the onset pattern of severe diabetic retinopathy. Nitric Oxide 13: 88-92.

44. Zanchi A, Moczulski DK, Hanna LS, Wantman M, Warram J, et al. (2000) Risk of advanced diabetic nephropathy in type 1 diabetes is associated with endothelial nitric oxide synthase gene polymorphism. Kidney Int 57: 405-413.

45. Mamoulakis D, Bitsori M, Galanakis E, Vazgiourakis V, Panierakis C (2009) Intron 4 polymorphism of the endothelial nitric oxide synthase eNOS gene and early microangiopathy in type 1 diabetes. Int J Immunogenet 36: 153-157.

46. Zintzaras E, Papathanasiou AA, Stefanidis I (2009) Endothelial nitric oxide synthase gene polymorphisms and diabetic nephropathy: a HuGE review and meta-analysis. Genet Med 11: 695-706.

47. Costacou T, Chang Y, Ferrell RE, Orchard TJ (2006) Identifying genetic susceptibilities to diabetes-related complications among individuals at low risk of complications: An application of tree-structured survival analysis. Am J of Epidemiology 164: 862-872.

48. Brouet A, Sonveaux P, Dessy C, Balligand JL, Feron O (2001) Hsp90 ensure the transition from the early $\mathrm{Ca} 2+$-dependent to the late phosphorylationdependent activation of the endothelial nitric oxide synthase in vascular endothelial growth factor-exposed endothelial cells. J Biol Chem 276: 3266332669 .

49. Bazzaz JT, Amoli MM, Pravica V, Chandrasecaran R, Boulton AJ, et al. (2010) eNOS gene polymorphism association with retinopathy in type 1 diabetes. Ophthalmic Genet 31: 103-107.

50. Taverna MJ, Sola A, Guyot-Argenton C, Pacher N, Bruzzo F, et al. (2002) eNOS4 polymorphism of the endothelial nitric oxide synthase predicts risk for severe diabetic retinopathy. Diabet Med 19: 240-245.

51. Mehrab-Mohseni M, Tabatabaei-Malazy O, Hasani-Ranjbar S, Amiri P Kouroshnia A, et al. (2011) Endothelial nitric oxide synthase VNTR (intron $4 \mathrm{a} / \mathrm{b}$ ) polymorphism association with type 2 diabetes and its chronic complications. Diabetes Res and Clin Pract 91: 348-352.

52. Wang J, Dudley D, Wang XL (2002) Haplotype-specific effects on endothelia NO synthase promoter efficiency: modifiable by cigarette smoking. Arterioscler Thromb Vasc Biol 22: e1-e4.

53. Ezzidi I, Mtiraoui N, Mohamed MBH, Mahjoub T, Kacem M, et al. (2008) Endothelial nitric oxide synthase Glu298Asp, 4b/a, and T-786C polymorphisms in type 2 diabetic retinopathy. Clin Endocrinol (Oxf) 68: 542-546. 